\title{
Ethnic variation in risk genotypes based on single nucleotide polymorphisms (SNPs) of the interferon-inducible transmembrane 3 (IFITM3) gene, a susceptibility factor for pandemic 2009 H1N1 influenza $A$ virus
}

\author{
Yong-Chan Kim ${ }^{1,2} \cdot$ Byung-Hoon Jeong ${ }^{1,2}$ (B)
}

Received: 19 September 2020 / Accepted: 3 November 2020 / Published online: 10 November 2020

(c) Springer-Verlag GmbH Germany, part of Springer Nature 2020

\begin{abstract}
The interferon-inducible transmembrane 3 (IFITM3) protein is an effector of the host innate immune system that shows defensive activity against a wide range of viruses, including the influenza A virus. Previous studies have reported that three transcription-related regulatory single nucleotide polymorphisms (SNPs), rs12252, rs34481144, and rs6598045, showed potent associations with the severity of pandemic influenza A 2009 infection and susceptibility to this virus, respectively. However, the distribution of the risk genotypes of these three SNPs according to ethnic background has remained elusive. In this study, we compared the genotype and allele frequencies of the IFITM3 polymorphisms among several ethnic groups including American, African, European, South Asian, and East Asian using chi-square test. In addition, we analyzed the worldwide distribution of risk genotypes for pandemic influenza A 2009 virus infection. We found that the genotype and allele distributions of the rs12252, rs34481144, and rs6598045 SNPs were significantly different among several ethnic groups. In addition, the risk genotypes of the IFITM3 polymorphisms were also significantly different worldwide. To the best of our knowledge, this was the first simultaneous estimation of the risk genotypes of the IFITM3 gene with respect to pandemic influenza A 2009 virus infection.
\end{abstract}

Keywords IFITM3 · Interferon · SNP · rs12252 - rs34481144 · rs6598045 · Promoter region, pandemic influenza A 2009

\section{Introduction}

The interferon-inducible transmembrane 3 (IFITM3) protein is a potent antiviral effector encoded by the IFITM3 gene, which is classified as a small interferon-stimulated gene (Diamond and Farzan 2013; Feeley et al. 2011; Kim and Jeong 2017; Shi et al 2017; Zani and Yount 2018). The IFITM3 protein protects hosts from viral invasion by prohibiting hemifusion between host cells and various viruses, including the influenza A virus (Bailey et al. 2014; Brass et al. 2009; Li et al. 2013). The length of the human IFITM3

Byung-Hoon Jeong

bhjeong@jbnu.ac.kr

1 Korea Zoonosis Research Institute, Jeonbuk National University, Iksan, Jeonbuk 54531, Republic of Korea

2 Department of Bioactive Material Sciences and Institute for Molecular Biology and Genetics, Jeonbuk National University, Jeonju, Jeonbuk 54896, Republic of Korea protein is relatively short (133 aa), and the IFITM3 protein contains an interspecific well-conserved CD225 domain (John et al. 2013). A previous study reported that ablation of the IFITM3 gene results in severe pneumonia in mildly influenza-infected mice and that elevated expression of the IFITM3 protein provides a dramatic increase in the survival rate of severely influenza-infected mice. These studies indicated that the expression level or integrity of IFITM3 protein can modulate the defensive capacity of the host immune system (Everitt et al. 2012). Thus, polymorphisms that can modulate the expression level or integrity of the IFITM3 protein are considered to be crucial factors in antiviral capacity. In addition, recent studies provide evidences that IFITM3 protein is also related to host adaptive immunity (Yánez et al. 2020). The expression of IFITM3 protein is regulated by $\mathrm{T}$ cell receptor (TCR) signal pathway and plays an essential role in T-helper Th1/Th2 differentiation (Bedford et al 2019; Wakim et al 2013; Yánez et al. 2019). Furthermore, IFITM3 protein is associated with antibody 
response to flu vaccine and prevents cells from infection (Lei et al 2020).

To date, nonsynonymous single nucleotide polymorphisms (SNPs) in the coding sequence (CDS) of the human IFITM3 protein that can induce amino acid sequence variation have not been identified. However, the rs 12252 SNP-C allele-inducing splicing variant of the human IFITM3 protein denotes reduced antiviral capacity against pandemic influenza A 2009 virus infection (Everitt et al. 2012; Prabhu et al. 2018; Xuan et al. 2015; Zhang et al. 2013). In addition, recent studies have reported that two regulatory SNPs, rs34481144 and rs6598045, located in the promoter region regulate promoter activity by altering the binding affinity of the transcription factors CTCF and TFII-I, respectively. These two regulatory SNPs affected the transcriptional activity of the IFITM3 gene and showed an association with the severity and susceptibility of pandemic influenza A 2009 virus infection (Allen et al. 2017; Kim et al. 2020). The mechanism of action (MOA) and the association of three SNPs with pandemic influenza A 2009 virus infection were still unclear, and the difference of genotype and allele distributions of the IFITM3 polymorphisms according to ethnic background is also still elusive thus far.

In the present study, we compared the ethnic distribution of the genotype and allele frequencies of the rs12252, rs34481144, and rs6598045 SNPs of the IFITM3 gene involved in pandemic influenza A 2009 virus infection using data from the 1000 Genomes Project and a previously reported population. In addition, we investigated the difference of the risk genotype distributions of pandemic influenza A 2009 virus infection in each ethnic group.

\section{Materials and methods}

\section{Data collection}

The genotype frequencies of rs12252, rs34481144, and rs6598045 SNPs of the IFITM3 gene in the Korean population were obtained from previous studies (Kim and Jeong 2017; Kim et al. 2020). The genotype distributions of these three SNPs were also collected from the 1000 Genomes Project.

\section{Statistical analysis}

Genotype and allele frequencies of the IFITM3 polymorphisms were compared by the chi-square test using SAS 9.4 Software (SAS Institute Inc., Cary, NC, USA).

\section{Results}

\section{Locations of the rs12252, rs34481144, and rs6598045 SNPs}

The locations of the rs12252 SNP (c.42T >C), rs34481144 SNP (c.-23G >A), and rs6598045 SNP (c.-188T>C) are
Fig. 1 Locations of single nucleotide polymorphisms (SNPs) of the IFITM3 gene. The black box indicates the coding sequence (CDS) of the IFITM 3 gene. The white box indicates the $5^{\prime}$ untranslated region (UTR) of the IFITM3 gene

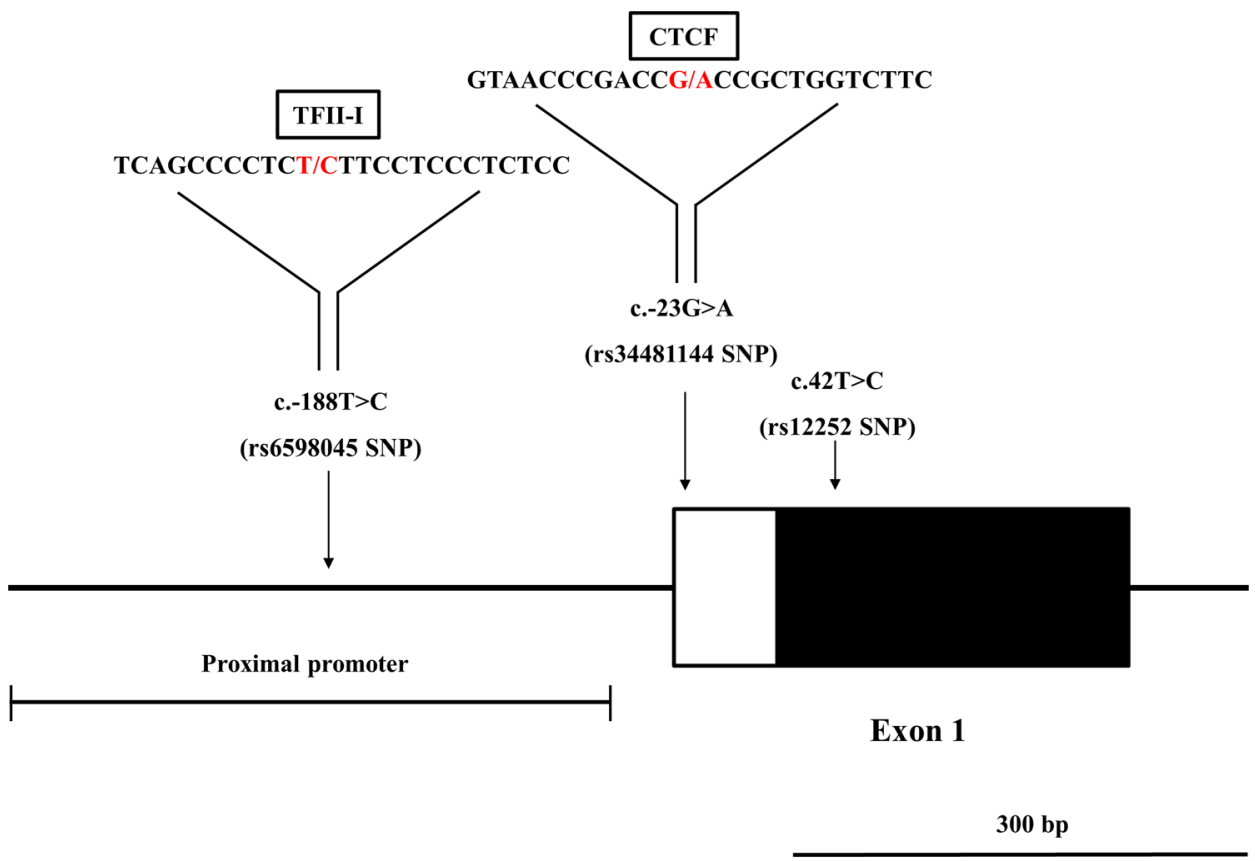


shown in Fig. 1. In brief, the rs 12252 SNP was located in the CDS, the rs34481144 SNP was located in the 5' untranslated region (UTR) of exon 1, and the rs6598045 SNP was located in the proximal promoter of the IFITM3 gene. The rs34481144 SNP is a transcription binding site of CTCF, and the rs6598045 SNP is a transcription binding site of TFII-I. Previous studies have reported that the binding ability of these transcription factors was modulated by the rs 34481144 and rs6598045 SNPs (Allen et al. 2017; Kim et al. 2020).

\section{Comparison of genotype and allele frequencies of the IFITM3 polymorphisms among several ethnic groups}

We compared the genotype and allele frequencies of the IFITM3 polymorphisms, including rs 12252, rs34481144, and rs6598045 SNPs among several ethnic groups. Detailed values are described in Tables 1 and 2. In brief, compared to rs12252, rs34481144, and rs6598045 SNPs of East Asian, those of the American, African, European, and South Asian showed significantly different distribution $(P<0.05)$. In Korean, compared to East Asian, except for rs34481144 SNP, the genotype and allele frequencies of rs12252 and rs34481144 SNPs showed significantly different distribution $(P<0.05)$.

\section{Distributions of risk genotypes for pandemic influenza A 2009 virus infection in several ethnic groups}

We also investigated risk genotypes with respect to pandemic influenza A 2009 virus infection. In brief, the risk genotype of the rs12252 SNP (CC genotype) was most frequently observed in East Asian populations (30\%). However, European (0\%), American (3\%), and South Asian populations (3\%) showed very low frequencies of the risk genotype of this SNP (Fig. 2). In addition, the risk genotype of the rs34481144 SNP (AA genotype) showed significantly different distributions among ethnic groups (Fig. 3). The risk genotype of rs34481144 was most frequently observed in European populations (22\%). However, Korean, East Asian, and African populations did not carry the risk genotype of this SNP. Furthermore, the risk genotype of the rs6598045 SNP was most frequently observed in European populations (79\%). However, compared to the European population, the African (46\%) and South Asian populations (49\%) showed lower frequencies of the risk genotype of this SNP (Fig. 4).
Table 1 Comparison of genotype frequencies of single nucleotide polymorphisms (SNPs) in the interferoninduced transmembrane protein gene (IFITM3) among several populations

\begin{tabular}{lllllll}
\hline Polymorphisms & Populations & $\begin{array}{l}\text { Total, } \\
n\end{array}$ & $\begin{array}{l}\text { Genotype } \\
\text { frequencies }\end{array}$ & $P$ value & Reference \\
\hline \multirow{2}{*}{ Rs12252 SNP } & & TT & TC & CC & & \\
& American & 235 & 101 & 11 & $<0.0001$ & 1000 genome project \\
& African & 363 & 252 & 46 & $<0.0001$ & 1000 genome project \\
& European & 462 & 41 & 0 & $<0.0001$ & 1000 genome project \\
& South Asian & 360 & 114 & 15 & $<0.0001$ & 1000 genome project \\
& East Asian & 123 & 230 & 151 & - & 1000 genome project \\
& Korean & 61 & 103 & 41 & 0.0223 & Kim and Jeong 2017; Kim et al 2020 \\
& & GG & GA & AA & & \\
& American & 213 & 106 & 28 & $<0.0001$ & 1000 genome project \\
& African & 605 & 55 & 1 & $<0.0001$ & 1000 genome project \\
& European & 148 & 245 & 110 & $<0.0001$ & 1000 genome project \\
& South Asian & 305 & 166 & 18 & $<0.0001$ & 1000 genome project \\
& East Asian & 498 & 6 & 0 & - & 1000 genome project \\
& Korean & 205 & 0 & 0 & 0.1893 & Kim and Jeong 2017; Kim et al 2020 \\
& & TT & TC & CC & & \\
& American & 199 & 142 & 6 & $<0.0001$ & 1000 genome project \\
& African & 305 & 317 & 39 & $<0.0001$ & 1000 genome project \\
& European & 400 & 99 & 4 & 0.0089 & 1000 genome project \\
& South Asian & 239 & 229 & 21 & $<0.0001$ & 1000 genome project \\
& East Asian & 364 & 138 & 2 & - & 1000 genome project \\
& Korean & 124 & 74 & 7 & 0.0002 & Kim and Jeong 2017; Kim et al 2020
\end{tabular}

Italicized texts indicate statistical significance $(P<0.05)$ 
Table 2 Comparison of allele frequencies of single nucleotide polymorphisms (SNPs) in the interferoninduced transmembrane protein gene (IFITM3) among several populations
Fig. 2 Worldwide distribution of the risk genotype (CC genotype) of the rs12252 SNP of the IFITM3 gene

\begin{tabular}{llllll}
\hline Polymorphisms & Populations & $\begin{array}{c}\text { Total, } n \\
\text { Rs12252 SNP }\end{array}$ & $\begin{array}{l}\text { Allele fre- } \\
\text { quencies }\end{array}$ & $P$ value & Reference \\
& American & T & C & & \\
& African & 571 & 123 & $<0.0001$ & 1000 genome project \\
& European & 978 & 344 & $<0.0001$ & 1000 genome project \\
& South Asian & 965 & 41 & $<0.0001$ & 1000 genome project \\
& East Asian & 834 & 144 & $<0.0001$ & 1000 genome project \\
Rs34481144 SNP & 476 & 532 & - & 1000 genome project \\
& Korean & 225 & 185 & 0.0089 & Kim and Jeong 2017; Kim et al 2020 \\
& American & $\mathrm{G}$ & $\mathrm{A}$ & & \\
& African & 532 & 162 & $<0.0001$ & 1000 genome project \\
& European & 1265 & 57 & $<0.0001$ & 1000 genome project \\
& South Asian & 541 & 465 & $<0.0001$ & 1000 genome project \\
& East Asian & 776 & 202 & $<0.0001$ & 1000 genome project \\
& Korean & 1002 & 6 & - & 1000 genome project \\
Rs6598045 SNP & 410 & 0 & 0.1903 & Kim and Jeong 2017; Kim et al 2020 \\
& American & T & $\mathrm{C}$ & & \\
& African & 540 & 154 & $<0.0001$ & 1000 genome project \\
& European & 927 & 395 & $<0.0001$ & 1000 genome project \\
& South Asian & 899 & 107 & 0.0186 & 1000 genome project \\
& East Asian & 707 & 271 & $<0.0001$ & 1000 genome project \\
& Korean & 866 & 142 & - & 1000 genome project \\
& & 322 & 88 & 0.0006 & Kim and Jeong 2017; Kim et al 2020
\end{tabular}

Italicized texts indicate statistical significance $(P<0.05)$

\section{Rs12252 SNP}

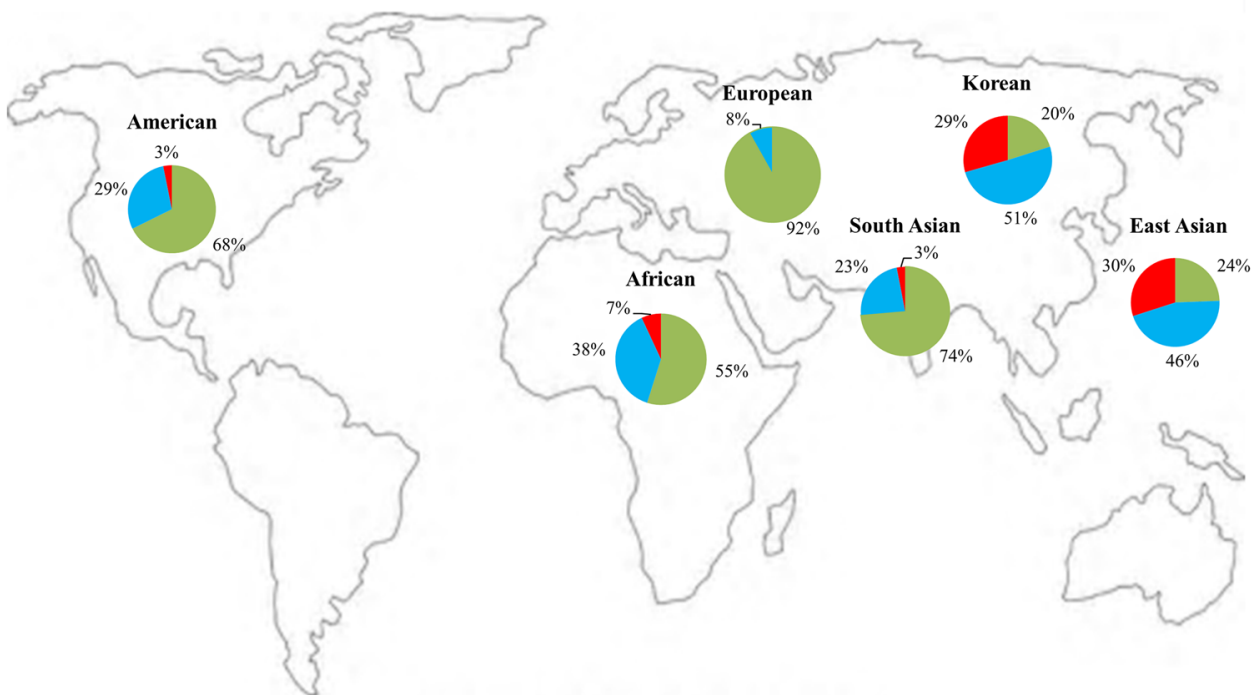

CC genotype (risk genotype)

CT genotype

TT genotype 
Fig. 3 Worldwide distribution of the risk genotype (AA genotype) of the rs34481144 SNP of the IFITM3 gene

\section{Rs34481144 SNP}

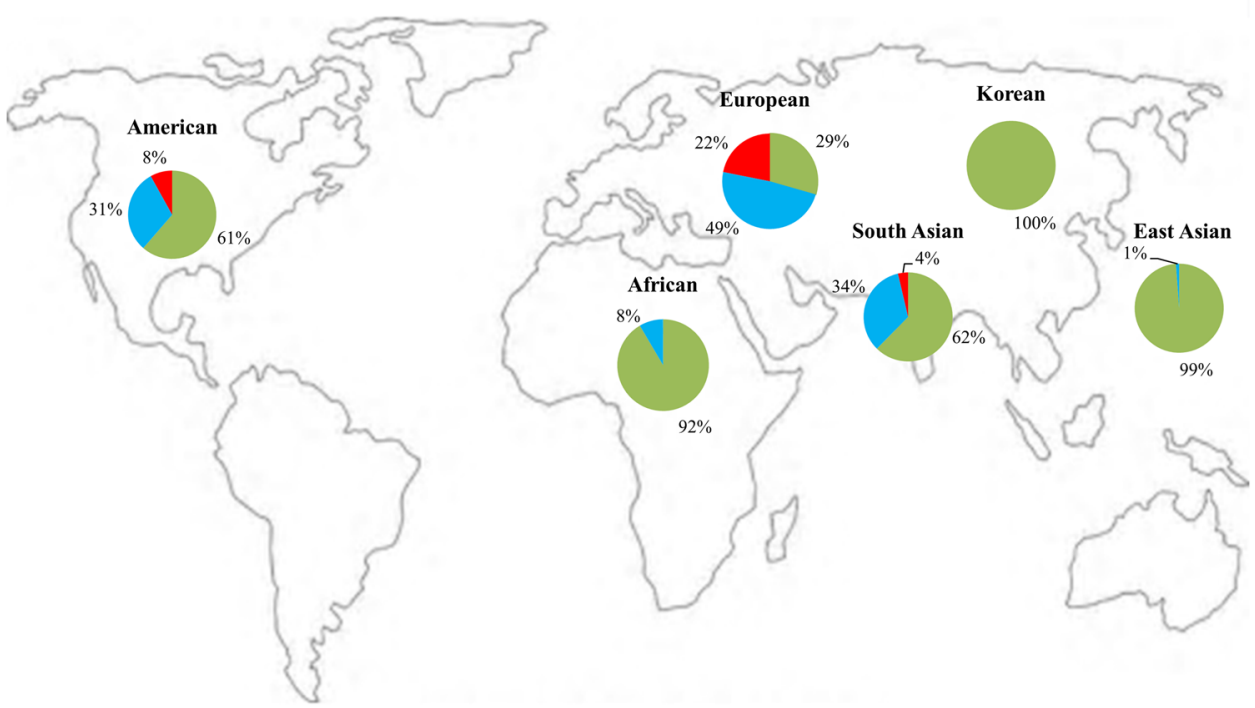

AA genotype (risk genotype)

AG genotype

GG genotype
Fig. 4 Worldwide distribution of the risk genotype (TT genotype) of the rs6598045 SNP of the IFITM3 gene

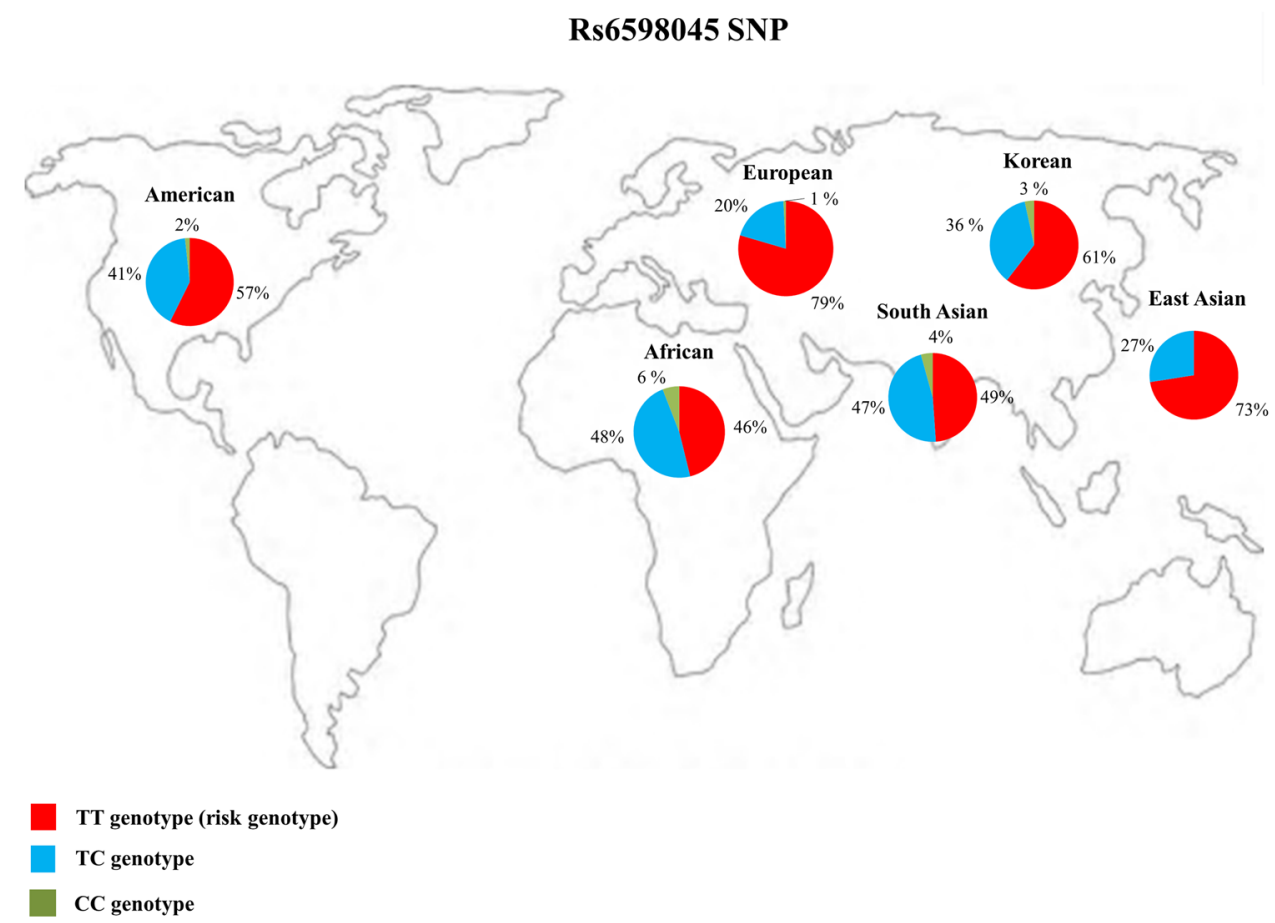




\section{Discussion}

The IFITM3 gene is classified as an interferon-stimulated gene (ISG) and a member of the IFITM protein family. The IFITM3 protein showed potent antiviral capacity against not only influenza A viruses but also Ebola virus (EBOV), Marburg virus (MARV), severe acute respiratory syndrome coronavirus (SARS-CoV), dengue virus (DEV), West Nile virus (WNV), and Zika virus (ZIKV) (Perreira et al. 2013; Zani and Yount 2018). Since the IFITM3 protein plays a pivotal role in blocking the invasion of several viruses, polymorphism studies that affect protein integrity or expression levels have received attention. Previous case-control studies and meta-analyses have confirmed the association of the rs12252 SNP with the severity of pandemic influenza A 2009 virus infection (Chen et al. 2018; Prabhu et al. 2018; Xuan et al. 2015; Yang et al. 2015). However, a study in a Korean population did not confirm the correlation of the disease severity of pandemic influenza A 2009 virus infection with the rs12252 SNP of the IFITM3 gene (Kim and Jeong 2017). In addition, the European population did not carry the risk genotype of the rs12252 SNP. Since these studies did not include correlation analysis of the worldwide distribution of pandemic influenza A 2009 virus-infected patients, a follow-up study suggested the rs34481144 SNP as a novel risk factor for pandemic influenza A 2009 virus infection. The rs34481144 SNP is located in a CpG island, and the A allele of the rs34481144 SNP disrupts the binding site of CTCF. In addition, the rs34481144 SNP acts as not only a proximal promoter of the IFITM3 gene but also a distal promoter of adjacent genes, including IFITM1, IFITM2, and B4GALNT4. These results indicate that the rs 34481144 SNP is an important site and regulates cascade-like IFITM3-related immune signaling. However, the Korean population did not harbor the rs34481144 SNP (Allen et al. 2017). In a recent study, the genotype of the rs6598045 SNP, which is located in the proximal promoter of the IFITM3 gene, modulated the transcriptional activity of the IFITM3 gene and was significantly associated with susceptibility to pandemic influenza A 2009 virus infection in the Korean population (Kim et al. 2020 ). Although the rs12252, rs34481144, and rs6598045 SNPs are related to the severity of pandemic influenza A 2009 infection or susceptibility to this virus, the ethnic distribution of the risk genotypes of these three SNPs has not been summarized thus far.

In the present study, we found significant difference of the genotype and allele frequencies of the IFITM3 polymorphisms among several ethnic groups. In addition, we summarized ethnic variations in the risk genotypes of the rs 12252, rs34481144, and rs6598045 SNPs. Interestingly, the European, American, and South Asian populations showed very low frequencies of the risk genotype of the rs12252 SNP (Fig. 2). In addition, the Korean, East Asian, and African populations did not carry the risk genotype of the rs34481144 SNP (Fig. 3). Furthermore, the risk genotype of the rs6598045 SNP showed variation among ethnic groups (Fig. 4). Since no single SNP could fully explain the severity or susceptibility of pandemic influenza A 2009 virus infection, evaluation of susceptibility based on haplotypes of the IFITM3 polymorphisms is highly desirable in the future. In addition, a recent study has been reported that rs 12252 SNP is associated with severity of coronavirus disease 2019 (COVID-19) (Zhang et al 2020). Thus, evaluation on an association between IFITM3 SNPs and susceptibility or severity of COVID-19 is needed in the future.

Since the IFITM3 protein has a highly homologous CD225 domain and is conserved among several species, it is necessary to investigate it in several viral host animals. In primate genome, IFITM3 protein is the most ancient antiviral protein of the IFITM locus and has undergone repeated duplication in each independent lineage. In addition, some IFITM3 genes in non-human primates have amino-terminal variations that alter protein localization and function (Compton et al 2016). A recent study reported a difference in the expression level of IFITM3 protein between avian influenza-susceptible animals and avian influenza-resistant animals (Smith et al. 2015). In addition, a difference in the genetic distribution of polymorphisms of the chicken IFITM3 gene was also identified between layers and broilers, which are bred for eggs and meats, respectively (Kim et al. 2019, 2020 ). Furthermore, since pig IFITM3 protein showed a defensive capacity against classical swine fever virus (CSFV), African swine fever virus (ASFV), and foot and mouth disease virus (FMDV), further investigation of the polymorphisms of animal IFITM3 genes is needed in the future ( $\mathrm{Li}$ et al. 2019; Munoz-Moreno et al. 2016; Xu et al. 2014).

In conclusion, we investigated the ethnic distribution of the risk genotypes of the rs12252, rs34481144, and rs6598045 SNPs with respect to pandemic influenza A 2009 virus infection. We observed significant differences in these three SNPs among the ethnic groups. In addition, we investigated the distribution of risk genotypes for pandemic influenza A 2009 virus infection. To the best of our knowledge, this is the first report of simultaneous evaluation of risk genotypes of the IFITM 3 gene with respect to pandemic influenza A 2009 virus infection.

Author contributions YCK and BHJ conceived and designed the experiment. YCK performed the experiments. YCK and BHJ analyzed the data. YCK and BHJ wrote the paper. All authors read and approved the final manuscript. 
Funding This research was supported by the Basic Science Program through the National Research Foundation (NRF) of Korea funded by the Ministry of Education, Science and Technology (2018R1D1A1B07048711). This research was supported by the Basic Science Research Program through the National Research Foundation (NRF) of Korea funded by the Ministry of Education (2017R1A6A1A03015876). This work was supported by NRF (National Research Foundation of Korea) Grant funded by the Korean Government (NRF-2019-Fostering Core Leaders of the Future Basic Science Program/Global Ph.D. Fellowship Program). Yong-Chan Kim was supported by the BK21 Plus Program in the Department of Bioactive Material Sciences.

Data availability All data generated or analyzed during this study are available from the corresponding author on reasonable request.

\section{Compliance with ethical standards}

Conflict of interest The authors declare that they have no conflict of interest.

\section{References}

Allen EK et al (2017) SNP-mediated disruption of CTCF binding at the IFITM3 promoter is associated with risk of severe influenza in humans. Nat Med 23:975-983. https://doi.org/10.1038/nm.4370

Bailey CC, Zhong G, Huang IC, Farzan M (2014) IFITM-family proteins: the cell's first line of antiviral defense. Annu Rev Virol 1:261283. https://doi.org/10.1146/annurev-virology-031413-085537

Bedford JG et al (2019) Rapid interferon independent expression of IFITM3 following T cell activation protects cells from influenza virus infection. PLoS ONE 14:e0210132. https://doi.org/10.1371/ journal.pone. 0210132

Brass AL et al (2009) The IFITM proteins mediate cellular resistance to influenza A H1N1 virus. Cell 139:1243-1254. https://doi. org/10.1016/j.cell.2009.12.017

Chen T, Xiao M, Yang J, Chen YK, Bai T, Tang XJ, Shu YL (2018) Association between rs12252 and influenza susceptibility and severity: an updated meta-analysis. Epidemiol Infect:1-9 https:// doi.org/10.1017/S0950268818002832

Compton AA et al (2016) Natural mutations in IFITM3 modulate posttranslational regulation and toggle antiviral specificity. EMBO Rep 17:1657-1671. https://doi.org/10.15252/embr.201642771

Diamond MS, Farzan M (2013) The broad-spectrum antiviral functions of IFIT and IFITM proteins. Nat Rev Immunol 13:46-57. https://doi. org/10.1038/nri3344

Everitt AR et al (2012) IFITM3 restricts the morbidity and mortality associated with influenza. Nature 484:519-523. https://doi. org/10.1038/nature10921

Feeley EM et al (2011) IFITM3 inhibits influenza A virus infection by preventing cytosolic entry. PLoS Pathog 7:e1002337. https://doi. org/10.1371/journal.ppat.1002337

John SP et al (2013) The CD225 domain of IFITM3 is required for both IFITM protein association and inhibition of influenza A virus and dengue virus replication. J Virol 87:7837-7852. https://doi. org/10.1128/JVI.00481-13

Kim YC, Jeong BH (2017) no correlation of the disease severity of influenza A virus infection with the rs 12252 polymorphism of the interferon-induced transmembrane protein 3 gene. Intervirology 60:69-74. https://doi.org/10.1159/000479087

Kim YC, Jeong MJ, Jeong BH (2019) Genetic characteristics and polymorphisms in the chicken interferon-induced transmembrane protein (IFITM3) gene. Vet Res Commun 43:203-214. https:// doi.org/10.1007/s11259-019-09762-y

Kim YC, Jeong MJ, Jeong BH (2020) Strong association of regulatory single nucleotide polymorphisms (SNPs) of the IFITM3 gene with influenza H1N1 2009 pandemic virus infection. Cell Mol Immunol 17:662-664. https://doi.org/10.1038/s41423-019-0322-1

Lei $\mathrm{N}$ et al (2020) IFITM3 affects the level of antibody response after influenza vaccination. Emerg Microbes Infect 9:976-987. https://doi. org/10.1080/22221751.2020.1756696

Li C, Zheng H, Wang Y, Dong W, Liu Y, Zhang L, Zhang Y (2019) Antiviral role of IFITM proteins in classical swine fever virus infection. Viruses 11. https://doi.org/10.3390/v11020126

$\mathrm{Li} \mathrm{K}$ et al (2013) IFITM proteins restrict viral membrane hemifusion. PLoS Pathog 9:e1003124. https://doi.org/10.1371/journal.ppat.1003124

Munoz-Moreno R et al (2016) Antiviral role of IFITM proteins in African swine fever virus infection. PLoS ONE 11:e0154366. https:// doi.org/10.1371/journal.pone.0154366

Perreira JM, Chin CR, Feeley EM, Brass AL (2013) IFITMs restrict the replication of multiple pathogenic viruses. J Mol Biol 425:49374955. https://doi.org/10.1016/j.jmb.2013.09.024

Prabhu SS, Chakraborty TT, Kumar N, Banerjee I (2018) Association between IFITM3 rs12252 polymorphism and influenza susceptibility and severity: a meta-analysis. Gene 674:70-79. https://doi. org/10.1016/j.gene.2018.06.070

Shi G, Schwartz O, Compton AA (2017) More than meets the I: the diverse antiviral and cellular functions of interferon-induced transmembrane proteins. Retrovirology 14:53. https://doi.org/10.1186/ s12977-017-0377-y

Smith J et al (2015) A comparative analysis of host responses to avian influenza infection in ducks and chickens highlights a role for the interferon-induced transmembrane proteins in viral resistance. BMC Genomics 16:574. https://doi.org/10.1186/s12864015-1778-8

Wakim LM et al (2013) Enhanced survival of lung tissue-resident memory $\mathrm{CD}^{+} \mathrm{T}$ cells during infection with influenza virus due to selective expression of IFITM3. Nat Immunol 14:238-245. https://doi.org/10.1038/ni.2525

$\mathrm{Xu} \mathrm{J}$ et al (2014) Swine interferon-induced transmembrane protein, sIFITM3, inhibits foot-and-mouth disease virus infection in vitro and in vivo. Antiviral Res 109:22-29. https://doi.org/10.1038/ s41589-018-0213-2

Xuan Y et al (2015) IFITM3 rs12252 T>C polymorphism is associated with the risk of severe influenza: a meta-analysis. Epidemiol Infect 143:2975-2984. https://doi.org/10.1017/S0950268815000278

Yánez et al (2019) IFITM proteins drive type $2 \mathrm{~T}$ helper cell differentiation and exacerbate allergic airway inflammation. European Journal of Immunology 49:66-78. https://doi.org/10.1002/ eji.201847692

Yánez DC, Ross S, Crompton T (2020) The IFITM protein family in adaptive immunity. Immunology 159:365-372. https://doi. org/10.1111/imm.13163

Yang X et al (2015) Interferon-inducible transmembrane protein 3 genetic variant rs 12252 and influenza susceptibility and severity: a meta-analysis. PLoS ONE 10:e0124985. https://doi.org/10.1371/ journal.pone.0124985

Zani A, Yount JS (2018) Antiviral protection by IFITM3 in vivo. Curr Clin Microbiol Rep 5:229-237. https://doi.org/10.1007/s40588-018-0103-0

Zhang $\mathrm{Y}$ et al (2020) Interferon-induced transmembrane protein-3 genetic variant rs 12252-C is associated with disease severity in COVID-19. J Infect Dis 222:34-37. https://doi.org/10.1093/infdis/jiaa224

Zhang YH et al (2013) Interferon-induced transmembrane protein-3 genetic variant rs12252-C is associated with severe influenza in Chinese individuals. Nat Commun 4:1418. https://doi. org/10.1038/ncomms2433 